\title{
Engobe borosilicatado como recubrimiento impermeable en mobiliario sanitario obtenido de altas concentraciones de ácido bórico residual
}

Bertha S. Vera-Barrios ${ }^{1 *}$ y Luis A. Lazo-Alarcón ${ }^{2}$

(1) Facultad de Ingeniería de Minas, Universidad Nacional de Moquegua, UNAM, Perú. Calle Ancash s/n. Mariscal Nieto Moquegua, Perú. (correo-e: silvanavera196812@gmail.com)

(2) Universidad Nacional de San Agustín, UNSA, Arequipa, Perú. (correo-e: luis8888lazo@gmail.com)

* Autor a quien debe ser dirigida la correspondencia

Recibido Dic. 27, 2019; Aceptado Feb. 28, 2020; Versión final Abr. 27, 2020, Publicado Ago. 2020

\section{Resumen}

El objetivo de este estudio es utilizar el ácido bórico residual como insumo para elaborar un esmalte vítreo altamente pulido e impermeable, es decir "baja absorción" que recubra mobiliario sanitario, permitiendo una rápida escorrentía de los residuos. Se elaboraron cuatro sub-tipos de mezcla, tres con concentraciones (10, 20 y $30 \%$ en peso) de ácido bórico respecto al total del peso del resto de insumos, y una mezcla para una muestra control sin incluir ácido bórico. Considerando como variables de estudio al "ácido bórico" y la "impermeabilidad", se validaron los resultados aplicando primeramente el modelo estadístico de un Anova unifactorial con bloques aleatorizado al azar, y luego la prueba estadística de T-Student. Los especímenes recubiertos de engobe borosilicatado con concentración del $20 \%$ en peso absorben menos agua que los demás especímenes en referencia. El estudio demuestra que es una alternativa ecologista para utilizarse en un futuro como recubrimiento altamente impermeable.

\section{Borosilicate clay as waterproof coating on sanitary furniture obtained from high concentrations of residual boric acid}

\begin{abstract}
The objective of this research study is to use residual boric acid as an input to make a highly polished and waterproof vitreous enamel that is "low absorption" and that is used to cover sanitary furniture for rapid waste runoff. Four sub-types of mixture were prepared: three with different concentrations $(10,20$, and $30 \%$ by weight) of boric acid with respect to the total weight of the rest of the inputs and one mixture for one control sample not including boric acid. Considering "boric acid" and "impermeability" as study variables, the results were validated by first applying the statistical model of a single-factor random block Anova followed by the statistical T-Student test. The specimens coated with $20 \%$ (by weight) borosilicate absorbed less water than the other reference specimens. This study demonstrates an environmentally friendly alternative for waterproof coating that can be used in the future.
\end{abstract}




\section{INTRODUCCION}

Los protocolos de seguridad ambiental del Ministerio de Energía y Minas (MINEM,2018), reportan que los plaguicidas elaborados con ácido bórico, luego de su utilización, dejan residuos boratados que se vierten en ríos y manantiales, los cuales al ser ingeridos tanto por el hombre y animales, ocasionan severos problemas de salud pública. La reutilización de estos compuestos boratados hoy en día, es materia de amplio estudio, para múltiples aplicaciones, de esta forma Hernández et al. (2019), demostraron que el ácido bórico puede formar compuestos boratados en combinación con la alúmina con adecuadas propiedades refractarias y de resistencia a la corrosión. Zanelli et al. (2019) menciona la utilidad que tienen los boratos residuales cuando se incorporan como materiales reciclables, demostrando así como aprovecharlos para fabricar baldosas cerámicas para pavimentos; estos mismos boratos también pueden reutilizarse como insumos alternativos en el diseño de compuestos vítreos con superficies altamente pulidas, herméticas y de reducida capacidad de absorción, que al ser utilizados como revestimientos en mobiliario sanitario, permitan una rápida escorrentía de residuos y así se reduce la cantidad de agua para la higiene respectiva.

Se han encontrado resultados de relevancia en publicaciones recientes sobre el tema de recubrimientos vítreos con inclusión de boro. Surendranathan et al. (2014), Varshneya y Mauro (2019), y Schoenfish et al. (2017), mencionan que al ser estos expuestos al agua, son afectados por un fenómeno corrosivo que altera su durabilidad. El vidrio, que es un compuesto vítreo, es una sustancia amorfa, como resultado de una transición acelerada de una fase liquida a una fase sólida, lo cual, ha impedido que sus estructuras moleculares atómicas e iónicas se dispongan en forma ordenada o siguiendo un patrón ordenado, diferenciándose de los sólidos cristalinos, los cuales se enfrían gradualmente y sus estructuras son ordenadas.

Sobre la importancia de la naturaleza de los materiales vítreos aplicados a biomateriales durante el proceso de solidificación. Saad (2019) enfatiza que es posible que tenga participación durante el proceso. Según la disposición de las moléculas de sílice $\left(\mathrm{SiO}_{2}\right)$, los compuestos pueden ser de estructura cristalina, (el cuarzo) o un sólido de estructura amorfa, (el vidrio). A partir de estos hallazgos, el producto desarrollado en este trabajo, presenta características y apariencia externa como sustancia amorfa, puesto que la textura de este engobe es la de una película delgada de consistencia vítrea. Así también Wang_et al. (2016) encuentran que el grafeno, utilizado como insumo para producir un recubrimiento delgado y transparente, tiene altas cualidades de impermeabilidad y resistencia química frente a fluidos como el agua, lo cual es un antecedente procedimental similar al desarrollado en esta investigación pero con diferente insumo. El comportamiento del engobe dentro de una pasta diseñada para recubrimientos, es el de una capa que se coloca sobre el soporte cerámico y precede al esmalte (Gomes et al., 2016; Cardona, 2013) y cuya presentación es la de una suspensión que incluye arcillas, minerales y defloculantes, con propósitos únicamente decorativos y/o de protección, por lo que no tiene en sí mismo, altas propiedades impermeables, y no se recomienda utilizarlo para estos fines.

En esta investigación, se utilizó al ácido bórico como insumo dentro del diseño de una pasta para elaborar "engobe borosilicatado" en altas concentraciones, el cual al desempeñar la función de fundente, permitió la vitrificación dando por resultado un compuesto, que podría utilizarse como recubrimiento, tanto en utensilios sanitarios, como en cualquier superficie que requiera utilizar agua para arrastrar residuos (Barba et al., 2002; Bruguera, 1985). Se experimentó utilizando distintas concentraciones de ácido bórico, permitiendo obtener varios prototipos de este engobe, con adecuada impermeabilidad y pulidez, observándose que se reduce notablemente la absorción en su superficie. Para generar las condiciones de impermeabilidad, se le ha incorporado ácido bórico hasta alcanzar un porcentaje del $30 \%$ del total del peso de todos los insumos de la pasta base, este incremento también permite bajar el punto de fusión, evitando utilizar altas temperaturas al momento de la cochura, (Aparicio y Galán, 2005).

\section{METODOLOGIA}

La Figura 1 de izquierda a derecha muestra: a) Procedimiento / inmersión; b) Vista lateral y vista superior de muestra dentro de recipiente con agua; c) muestra con engobe y muestra patrón; d) muestra y patrón dimensional $(\mathrm{cm})$, abajo garrafa con patrón dimensional $(\mathrm{cm})$ dispuesto horizontalmente y garrafa con patrón dimensional dispuesto verticalmente.

Dentro del conjunto de insumos conformantes se considera, además del ácido bórico, materiales como caolín, cuarzo, bórax y pigmento sintético y otros reciclados como vidrio molido y arcillas de piedras lajas molidas. La combinación de todos estos insumos da por resultado una mezcla de consistencia viscosa de color amarillo, la cual al recubrir un sustrato ya cocido de barbotina, se lleva al horno a $950^{\circ} \mathrm{C}$ y da por resultado una película vítrea brillante poco traslucida (Tabla 1). 


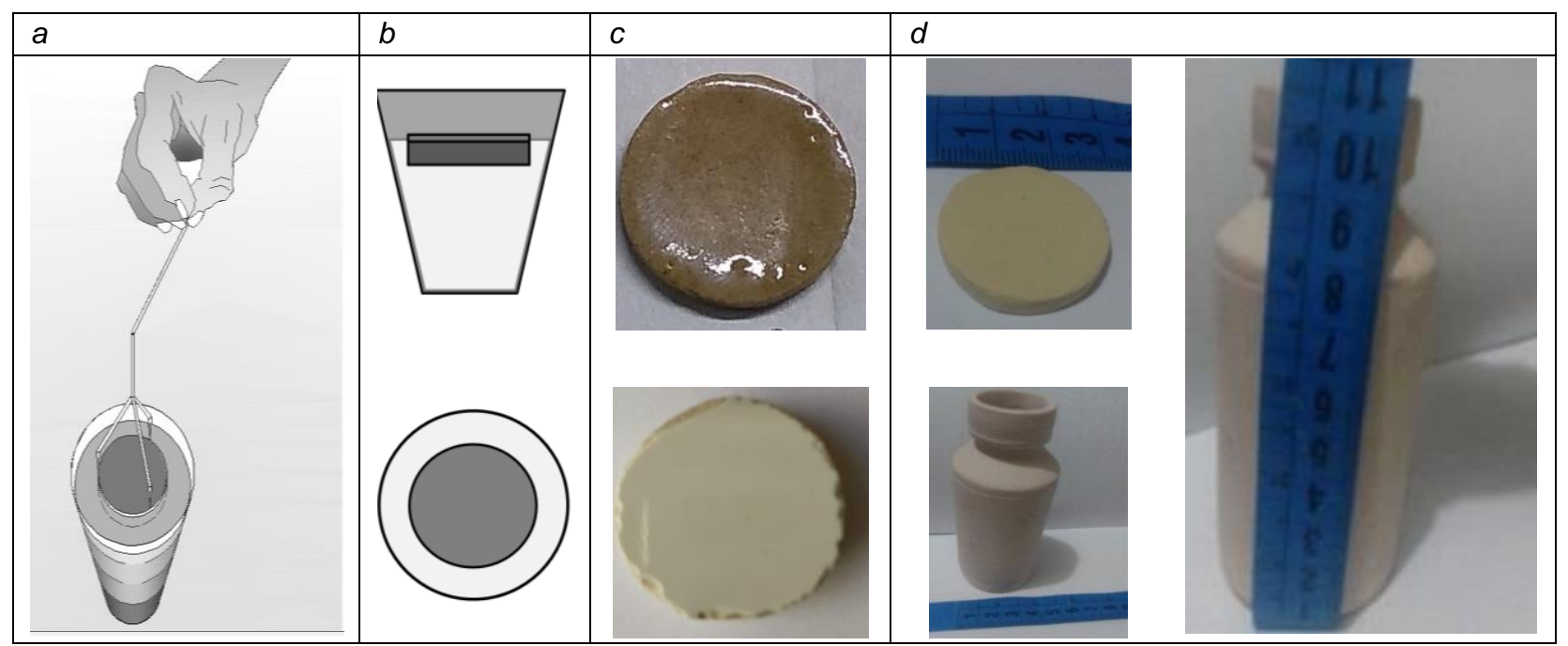

Fig.1: procedimiento de la norma ASTM C127-04 para la inmersión de las muestras en agua

Tabla 1: Materiales y equipos utilizados en el proyecto

\begin{tabular}{|l|l|l|l|l|l|}
\hline \multicolumn{2}{|l|}{$\begin{array}{l}\text { Materiales e Insumos utilizados para las } 04 \\
\text { experimentaciones }\end{array}$} & \multicolumn{2}{l|}{$\begin{array}{l}\text { Características físicas de las } \\
\text { muestras }\end{array}$} & \multicolumn{2}{l|}{ Equipos } \\
\hline 1) Arcillas de piedras lajas molidas & $50 \mathrm{~g}$ & Diámetro & $2.50 \mathrm{~cm}$ & Recipientes de Ágata & 15 uds. \\
\hline 2) Caolín & $120 \mathrm{~g}$ & Espesor & $0.55 \mathrm{~cm}$ & Vasos precipitados de vidrio & 25 uds \\
\hline 3) Bórax & $138 \mathrm{~g}$ & Peso & $3.80 \mathrm{gr}$ & Tamices normalizados/granulometría 200 & 08 uds. \\
\hline 4) Cuarzo & $40 \mathrm{~g}$ & Superficie a sumergir & $8.40 \mathrm{~cm}^{2}$ & Capsulas de porcelana de $50 \mathrm{~mm}$ & 25 uds. \\
\hline $\begin{array}{l}\text { 5) Vidrio molido malla pasante } \\
\text { malla } 200\end{array}$ & $18 \mathrm{~g}$ & Espesor de engobe & $0.5 \mathrm{~mm}$ - \\
\hline 6) Marmolina blanca & $30 \mathrm{~g}$ & & $\begin{array}{l}\text { Crisoles de porcelana y planchas } \\
\text { refractarias }\end{array}$ & 25 uds. \\
\hline 7) Ácido bórico & $60 \mathrm{~g}$ & & Compresora hidráulica & 02 uds. \\
\hline $\begin{array}{l}\text { 8) Pigmento Amarillo sintético y } \\
\text { pigmento blanco }\end{array}$ & $20 \mathrm{~g}$ & & & $\begin{array}{l}\text { Hascarillas, lentes y guantes protección } \\
\text { balanza de precisión }\end{array}$ & 24 uds. \\
\hline
\end{tabular}

Los insumos de los que está hecho, se encuentran organizados en 4 sub-grupos, a saber: "plastificantes", un $40 \%$ representado por las arcillas ya mencionados y caolín, "desengrasantes" un 10\% representado por cuarzo y vidrio molido, "fundentes" un 50\% representado por bórax y ácido bórico. Finalmente un $10 \%$ incluye defloculantes, el silicato de sodio, pigmentos sintéticos y agua (ver tablas 2 a 4). Para obtener un producto adecuado en el que tanto la barbotina o arcilla base, como el engobe se acoplen perfectamente, se debe evitar que se produzca la contracción, es decir se produzcan resquebrajaduras en el engobe por encogimiento, para ello se aplicó el engobe sobre la barbotina cuando la pasta estuvo como "bizcocho" y en similar temperatura que la del engobe, empleándose la técnica de aspersión con compresora para aplicar el engobe (Figura 2).

Como el estudio se centra específicamente en la variable "ácido bórico", el objetivo es determinar que porcentajes de ácido bórico que ingresa como insumo "fundente", dentro de la composición de varios prototipos de engobe sanitario de borosilicato, son los que dan por resultado un producto mejorado con menores niveles de absorción de agua para producir esmaltes impermeables recubridores de mobiliario sanitario. Por lo tanto se determinó a través de la utilización de un modelo estadístico Anova unifactorial con bloques completamente aleatorizado, luego de comparar 3 tipos de mezclas que contienen ácido bórico para engobe, cuál fue la menos absorbente o más impermeable, así se seleccionó a la que presentaba menos niveles de absorción, es decir la que tenga el mejor desempeño como esmalte impermeable, finalmente esta última, cuya concentración de ácido bórico es del $20 \%$, se comparó con la "muestra control" proveniente de un trozo de loza vitrificada con la que se fabrican los inodoros convencionales.

Los resultados evidenciaron que la muestra con concentración del $20 \%$ de ácido bórico, es la que absorbe menos agua. El análisis incluye dos partes, en la primera parte se expone las evidencias encontradas de la parte experimental realizada con los 3 sub-tipos de engobe, aplicando el modelo estadístico Anova unifactorial ya referido, para validar estos resultados, la segunda parte es aplicar el T - Student, para comparar los niveles de absorción de agua que se obtuvo del espécimen "muestra control" con los niveles de absorción de los especímenes de engobe borosilicatado, que tienen concentración de ácido bórico de $20 \%$. En la Figura 2 vemos el procedimiento para conseguir los resultados esperados. 

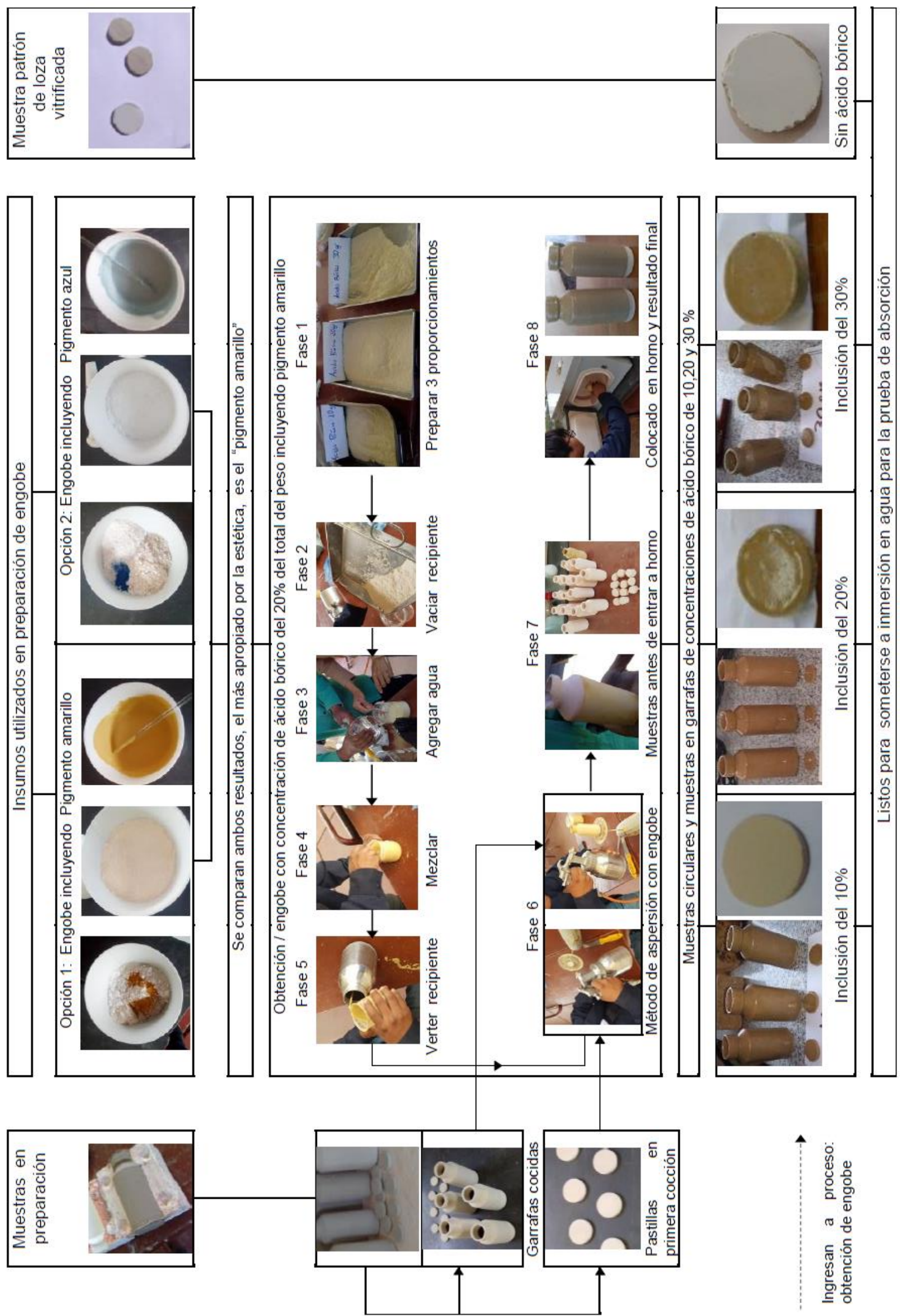

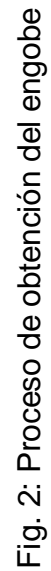


Tabla 2: Proporcionamiento incluyendo solo $10 \mathrm{~g}$ de ácido bórico o $10 \%$ en peso

\begin{tabular}{|c|c|c|c|c|c|c|c|c|c|c|c|}
\hline \multicolumn{12}{|c|}{ Grupo "1" Concentración de $10 \%$ de ácido bórico } \\
\hline & \multirow[t]{2}{*}{$100 \%$} & \multirow{2}{*}{$\begin{array}{l}\text { Arcillas de } \\
\text { piedras lajas } \\
\text { molidas }\end{array}$} & \multirow[t]{2}{*}{ caolín } & \multirow[t]{2}{*}{ bórax } & \multirow{2}{*}{$\begin{array}{l}\text { Ácido } \\
\text { bórico }\end{array}$} & \multirow[t]{2}{*}{ cuarzo } & \multirow{2}{*}{$\begin{array}{l}\text { Vidrio } \\
\text { molido }\end{array}$} & \multirow{2}{*}{$\begin{array}{r}\text { Silicato } \\
\text { sódico }\end{array}$} & \multicolumn{2}{|c|}{ Pigmento Sintéticos } & \multirow{2}{*}{$\begin{array}{l}\text { Peso } \\
\text { seco }\end{array}$} \\
\hline & & & & & & & & & $\begin{array}{l}\text { Pigmento } \\
\text { amarillo }\end{array}$ & $\begin{array}{l}\text { Marmolina } \\
\text { blanca }\end{array}$ & \\
\hline Plastificantes & $30 \%$ & $10 \mathrm{~g}$ & $20 \mathrm{~g}$ & - & - & - & - & opcional & & & 30 \\
\hline Fundentes & $50 \%$ & - & - & $40 \mathrm{~g}$ & $10 \mathrm{~g}$ & - & - & opcional & - & & 50 \\
\hline Desengrasantes & $10 \%$ & - & - & - & - & $4 \mathrm{~g}$ & $6 \mathrm{~g}$ & opcional & - & & 10 \\
\hline \multirow[t]{2}{*}{ Colorantes } & $10 \%$ & & & & & & & opcional & $0.6 \mathrm{~g}$ & $9.4 \mathrm{~g}$ & 10 \\
\hline & \multicolumn{10}{|c|}{ TOTAL } & $100 \mathrm{~g}$ \\
\hline
\end{tabular}

Tabla 3: Proporcionamiento incluyendo solo $20 \mathrm{~g}$ de ácido bórico o $20 \%$ en peso

\begin{tabular}{|c|c|c|c|c|c|c|c|c|c|c|c|}
\hline \multicolumn{12}{|c|}{ Grupo "2" Concentración de $20 \%$ de ácido bórico } \\
\hline & \multirow[t]{2}{*}{$100 \%$} & \multirow{2}{*}{$\begin{array}{l}\text { Arcillas de } \\
\text { piedras lajas } \\
\text { molidas }\end{array}$} & \multirow[t]{2}{*}{ caolín } & \multirow[t]{2}{*}{ bórax } & \multirow{2}{*}{$\begin{array}{l}\text { Ácido } \\
\text { bórico }\end{array}$} & \multirow[t]{2}{*}{ cuarzo } & \multirow{2}{*}{$\begin{array}{l}\text { Vidrio } \\
\text { molido }\end{array}$} & \multirow{2}{*}{$\begin{array}{r}\text { Silicato } \\
\text { sódico }\end{array}$} & \multicolumn{2}{|c|}{ Pigmento Sintéticos } & \multirow{2}{*}{$\begin{array}{l}\text { Peso } \\
\text { seco }\end{array}$} \\
\hline & & & & & & & & & $\begin{array}{l}\text { Pigmento } \\
\text { amarillo }\end{array}$ & $\begin{array}{l}\text { Marmolina } \\
\text { blanca }\end{array}$ & \\
\hline Plastificantes & $30 \%$ & $10 \mathrm{~g}$ & $20 \mathrm{~g}$ & - & - & - & - & opcional & & & 30 \\
\hline Fundentes & $50 \%$ & - & - & $330 \mathrm{~g}$ & $220 \mathrm{~g}$ & - & - & opcional & - & & 50 \\
\hline Desengrasantes & $10 \%$ & - & - & - & - & $4 g$ & $6 \mathrm{~g}$ & opcional & - & & 10 \\
\hline \multirow[t]{2}{*}{ Colorantes } & $10 \%$ & & & & & & & opcional & $0.6 \mathrm{~g}$ & $9.4 \mathrm{~g}$ & 10 \\
\hline & \multicolumn{10}{|c|}{ TOTAL } & $100 \mathrm{~g}$ \\
\hline
\end{tabular}

Tabla 4: Proporcionamiento incluyendo solo $30 \mathrm{~g}$ de ácido bórico o 30\% en peso

\begin{tabular}{|c|c|c|c|c|c|c|c|c|c|c|c|}
\hline \multicolumn{12}{|c|}{ Grupo "3" Concentración de $30 \%$ de ácido bórico } \\
\hline & \multirow[t]{2}{*}{$100 \%$} & \multirow{2}{*}{$\begin{array}{l}\text { Arcillas de } \\
\text { piedras } \\
\text { lajas } \\
\text { molidas }\end{array}$} & \multirow[t]{2}{*}{ caolín } & \multirow[t]{2}{*}{ bórax } & \multirow{2}{*}{$\begin{array}{l}\text { Ácido } \\
\text { bórico }\end{array}$} & \multirow[t]{2}{*}{ cuarzo } & \multirow{2}{*}{$\begin{array}{l}\text { Vidrio } \\
\text { molido }\end{array}$} & \multirow{2}{*}{$\begin{array}{l}\text { Silicato } \\
\text { sódico }\end{array}$} & \multicolumn{2}{|c|}{ Pigmento Sintéticos } & \multirow{2}{*}{$\begin{array}{l}\text { Peso } \\
\text { seco }\end{array}$} \\
\hline & & & & & & & & & $\begin{array}{l}\text { Pigmento } \\
\text { amarillo }\end{array}$ & $\begin{array}{l}\text { Marmolina } \\
\text { blanca }\end{array}$ & \\
\hline Plastificantes & $30 \%$ & $10 \mathrm{~g}$ & $20 \mathrm{~g}$ & - & - & - & - & opcional & & & 30 \\
\hline Fundentes & $50 \%$ & - & - & $20 \mathrm{~g}$ & $30 \mathrm{~g}$ & - & - & opcional & - & & 50 \\
\hline Desengrasantes & $10 \%$ & - & - & - & - & $4 \mathrm{~g}$ & $6 \mathrm{~g}$ & opcional & - & & 10 \\
\hline \multirow[t]{2}{*}{ Colorantes } & $10 \%$ & & & & & & & opcional & $0.6 \mathrm{~g}$ & $9.4 \mathrm{~g}$ & 10 \\
\hline & \multicolumn{10}{|c|}{ TOTAL } & $100 \mathrm{~g}$ \\
\hline
\end{tabular}

Tabla 5: Proporcionamiento de muestra patrón (tomado de las características del fabricante)

\begin{tabular}{|c|c|c|c|c|c|c|c|c|}
\hline \multicolumn{9}{|c|}{ Grupo "4": Sin Concentración de ácido bórico } \\
\hline & $100 \%$ & arcillas & caolín & bórax & cuarzo & Silicato sódico & Pigmento blanco u "otro" & Peso seco \\
\hline Plastificantes & $65 \%$ & $10 \mathrm{~g}$ & $55 \mathrm{~g}$ & & & opcional & & 65 \\
\hline Fundentes & $15 \%$ & & & $15 \mathrm{~g}$ & & opcional & & 15 \\
\hline Desengrasantes & $10 \%$ & & & & $10 \mathrm{~g}$ & opcional & & 10 \\
\hline \multirow[t]{2}{*}{ Colorantes } & $10 \%$ & & & & & opcional & $10 \mathrm{~g}$ & 10 \\
\hline & \multicolumn{7}{|c|}{ TOTAL } & $100 \mathrm{~g}$ \\
\hline
\end{tabular}

Los procedimientos y metodologías avanzados sobre el estudio de materiales vítreos, demandan técnicas estadísticas que agilicen el procesamiento de datos y resultados. En la investigación denominada Systematic study of sol-gel parameters on $\mathrm{TiO}_{2}$ coating for $\mathrm{CO}_{2}$ photoreduction (Thompson et al., 2018), que trata sobre recubrimientos, se evidencia la utilidad de los modelos estadísticos que utilizan la técnica estadística del ANOVA, la cual se empleó paralelamente con los diseños experimentales (DOE) y un uso eficiente de Plackett-Burman (PB), en ambos casos son asistidos por esta técnica, como método estadístico inicial para validar que no existen diferencias significativas en la comparación de 4 parámetros importantes, medias de banda prohibida, fase anatasa, fase rutilo y cristalinidad de la fase anatasa. 
El ANOVA, permitió reforzar que los resultados obtenidos de la aplicación del modelo PB, donde el volumen de PPG no tuvo un efecto estadísticamente significativo en estas respuestas. Este antecedente metodológico con resultados muy precisos para determinar diferencias significativas entre parámetros, es un referente para elegir el modelo experimental ANOVA unifactorial con bloques aleatorizados, siguiendo el procedimiento de Cruz (2015), para poder saber cuál es el efecto que tiene la variable explicativa (ácido bórico) en concentraciones distintas de 10, 20 y $30 \%$ en la variable dependiente impermeabilidad o menor absorción de agua, en comparación con una cuarta muestra que corresponde a una muestra patrón. (Figura 4) y Tabla 8 , utilizándose un prototipo de espécimen (Figura 1), que es una pastilla circular utilizada en los 4 grupos de análisis. A efecto de ello consideramos un total de 10 niveles (días) "bloques" con 3 tratamientos "diversos proporciones de ácido bórico", lo cual nos da un total de 29 unidades experimentales.

Con respecto a los diagramas de utilidad, en nuestro trabajo se ha utilizado los diagramas de Tukey, siguiendo apropiadamente los sistemas de bloques y los grupos respectivos organizándose en niveles y tratamientos (ver tablas 2 a 5 y tabla 9). Wandscher et al. (2016), emplearon esa misma metodología con el ANOVA de dos vías, trabajándose con una gran cantidad de datos asociada a la prueba de Tukey, lo cual permitió en dos oportunidades reafirmar los valores de cohesión y unión entre la circonia y los cemento de resina para mejorar las propiedades triboquimicas de un nuevo e innovador revestimiento cerámico de vidrio triboquímico.

El procedimiento para demostrar comparación de medias fue la prueba estadística del T-Student, identificando así que provienen de poblaciones independientes y normales. Con el ANOVA, la investigación busca saber cuál de los 4 proporcionamientos "3 con ácido bórico y una muestra control sin adición de ácido bórico", presenta el menor valor numérico en lo que respecta a la absorción, expresada mediante un "porcentaje" según la Norma ASTM C-127-15 (ver tablas 2 a 5 y Figura 1). La experimentación requirió de un total de 46 muestras, 10 en los grupos 1 y 2, 9 en el grupo 3 y 7 para el cuarto grupo, de las cuales 29 se tabularon para aplicar el método del ANOVA unifactorial con bloques completamente aleatorizado y 17 del total, para aplicar el T-Student. La experimentación tomo 20 días, de los cuales, los resultados correspondientes a los primeros 10 días consecutivos corresponden al análisis del ANOVA de la primera parte, (Figura 3). En el trabajo realizado por De Mello et al. (2016), que trata del mejoramiento de los recubrimientos o esmaltes dentales a partir de fosfato de caseína amorfo y fosfato de calcio para remineralizar superficies dañadas, realizo la distribución y agrupación de sus datos en 4 bloques por 5 tratamientos, utilizando el ANOVA de un solo factor, relacionando las variables de superficie de dureza, profundidad de lesión, perdida de superficie mineralizada y como tratamientos al placebo y fosfato de caseína amorfo en diversos tiempos entre 3 y 8 horas. Tomando como referencia esta metodología de análisis, también se ha hecho los agrupamientos (Figura 3) de 3 bloques y 10 tratamientos, de manera similar se utilizó una distribución de probabilidad del T- Student en una segunda etapa (ver tablas 11 y 12).

Finalmente se tomó como referencia el trabajo de investigación desarrollado por Mehta et al. (2017), en el cual se aplica los métodos estadísticos de diseños experimentales (DOE) de mucha utilidad para la temática de los recubrimientos vítreos y recubrimientos por pulverización térmica, en esta investigación también se utilizan materiales como el acero y el boro para darle mayor solidez al producto deseado, recomendándose el uso del ANOVA como complemento para manejar adecuadamente datos y resultados. En la prueba estadística del T-Student, se consideraron los otros 10 niveles organizados, (Figura 4) para construir el intervalo de confianza y la comparación de dos medias de poblaciones independientes y normales. Se consideró el efecto del factor tiempo "días", que se presenta en de diez niveles, sobre la variable respuesta. La preparación de las muestras se inició produciendo la pasta del engobe, posterior fabricación de las probetas para su cochura, sometimiento a cocción en horno hasta que se encuentren en estado de bizcocho, cubrirlas con este engobe y nuevamente llevar al horno durante 9 horas a una temperatura de $950^{\circ} \mathrm{C}$; una vez obtenido el vitrificado se sumergió cada una en agua durante 24 horas, (Figura 1) lo cual se hizo con cada una de las 46 probetas. En las Tablas 6 y 7 puede observarse los resultados de la absorción en cada grupo de muestras. La ecuación 1 muestra la absorción de agua; donde $\mathrm{B}$ es la masa húmida final ( $\mathrm{g}$ ) y A la masa seca $(g)$ de acuerdo a la Norma ASTM C-127-15.

$$
\text { Absorción, \% }=[(B-A) / A] \times 100
$$

\section{RESULTADOS}

En la Figura 3, se observan los primeros 3 grupos de resultados de absorción en función de la concentración de ácido bórico con diferentes valores por día de prueba, en la Figura 4, se aplica el mismo procedimiento pero comparando el resultado más óptimo obtenido en la Figura 3 con los resultados de la muestra patrón. En las Tablas 6 y 7 vemos con detalle los datos obtenidos del incremento en peso promedio luego de la inmersión en agua de todo los especímenes de prueba representativos, a saber de 10,20 y $30 \%$ de inclusión de ácido bórico así como de la muestra patrón. Siendo la variable independiente el ácido bórico encontramos 
que luego de aplicar el modelo estadístico del Anova unifactorial en bloques se encontró que el coeficiente de variación (CV) es del 7.8\%, como puede observarse en la ecuación 2 (ver tabla 8), demostrando que tiene un grado de variabilidad muy baja y consecuentemente la media general tiene buena representatividad para todo el diseño y en consecuencia el diseño realizado tiene muy buena confiabilidad.

$$
C V=\frac{\sqrt{0.000245}}{0.200552} \times 100=7.804 \%
$$

Tanto para los tratamientos como para los bloques las hipótesis estadísticas se formularon al $5 \%$ y en ambos casos (para grupos como para bloques o tiempo), los resultados son menores al $5 \%$ ( 0.000 y 0.011$)$, por tanto las hipótesis para estos casos queda confirmada concluyéndose que el porcentaje de ácido bórico influye en el grado de impermeabilidad de los engobes de borosilicato, existiendo diferencia entre los grupos; así también para los bloques, se encontró que el tiempo influye en los niveles de absorción de agua, es decir que el haber estratificado la experimentación en días, ha reducido el error experimental o que la precisión del experimento ha sido mejorado en relación al diseño completamente al azar. (Figura 3).

Según la tabla de comparaciones múltiples (ver tabla 9) se presentan los resultados de 4 pruebas, de Tukey, Scheffe, DMS y Bonferroni, lo cual demuestra que existe diferencias significativas entre los 3 primeros grupos, es así que existe diferencia significativa entre el grupo 1 y grupo 2 con un valor sig. de 0.000 , así también hay diferencia significativa entre el grupo 1 y el grupo 3 con un valor sig. de 0.000 y también hay diferencia significativa entre el grupo 2 y el grupo 3 , demostrando un valor sig. de 0.000 ; consecuentemente según las cuatro pruebas estadísticas aplicadas demuestran que hay diferencias significativas en sus comparaciones.

En la Figura 3 vemos un resumen de los resultados de la prueba de absorción, en la que el engobe del Grupo 2 es el que demostró tener menor valor numérico en los márgenes de absorción de agua. La Tabla 10 muestra la comparación de promedios entre los tres grupos, por lo tanto, se aprecia para el grupo uno, que tiene un promedio de 0.213 , representa un intermedio entre los grupos dos y tres, mientras que para el grupo dos se muestra un promedio de 0.078 cuyo valor promedio es el menor respecto a los grupos uno y tres, así también que para el grupo tres cuyo promedio es el más alto y cuyo valor es de 0.322 , demostrando así que dicho grupo representa el más alto.

En cuanto a los supuestos de normalidad (Figuras 5 y 7), para los 3 porcentajes de ácido bórico, se confirma la hipótesis planteada y contrastada sobre la normalidad de datos, lo cual es confirmado por el diagrama de cajas y bigotes (Figuras 6 y 8). En lo referente a la prueba estadística de T-Student, en cuanto a la comprobación del supuesto de normalidad, según la Tabla 11, observamos que para los dos grupos se muestran valores de 0,078 y de 0,120 , por tanto son mayores al 0.050 de $5 \%$, consecuentemente podemos afirmar que los datos (Niveles de absorción de agua) se distribuyen de forma normal, la figura de normalidad confirma la hipótesis planteada y contrastada sobre la normalidad de datos. (Figura 7).

En la Comprobación del supuesto de homocedasticidad, luego de observar que para los dos grupos muestran valores de 0,001 por tanto son menores al 0.050 de $5 \%$, consecuentemente afirmamos que las varianzas no son iguales para ambos grupos, y se trabajara cuando las varianzas de ambos grupos son diferentes. En la comprobación por diferencia de grupos, se puede advertir (ver tabla 11), que para varianzas diferentes, muestra un valor sig. de 0,041 , es menor al $5 \%$, consecuentemente podemos afirmar que existe diferencia en ambos grupos. Finalmente en los diagramas de cajas y bigotes ( Figura 6), observamos que la figura de diagrama de caja y bigotes muestra el comportamiento que confirma la hipótesis planteada y demostrada en la cual existe diferencias entre ambos grupos, es así que para el grupo de $20 \mathrm{~g}$ de ácido bórico en la mezcla base, en la cual muestra que existe pequeña dispersión en sus datos y es menor con respecto al nivel de absorción de $0 \mathrm{~g}$ de ácido bórico, además dicho nivel según el diagrama de cajas, se encuentra por encima del nivel de $20 \mathrm{~g}$ de ácido bórico, demostrando además que sus datos se encuentran ligeramente dispersos.

Tabla 6: Índices de absorción de agua promedio por cada grupo de análisis

\begin{tabular}{|l|l|l|l|}
\hline & Grupo 1 & Grupo 2 & Grupo 3 \\
\hline Superficie cubierta con engobe & $8.4 \mathrm{~cm}^{2}$ & $8.4 \mathrm{~cm}^{2}$ & $8.4 \mathrm{~cm}^{2}$ \\
\hline Peso promedio agua absorbida & $0.213 \%$ absorción & $0.078 \%$ absorción & $0.323 \%$ absorción \\
\hline
\end{tabular}

Tabla 7: Índices de absorción de agua promedio por cada grupo de análisis

\begin{tabular}{|l|l|l|}
\hline & Grupo 2 & Grupo 3 \\
\hline Superficie cubierta con engobe & $8.4 \mathrm{~cm}^{2}$ & $8.4 \mathrm{~cm}^{2}$ \\
\hline Peso promedio agua absorbida & $0.078 \%$ absorción & $0.120 \%$ absorción \\
\hline
\end{tabular}




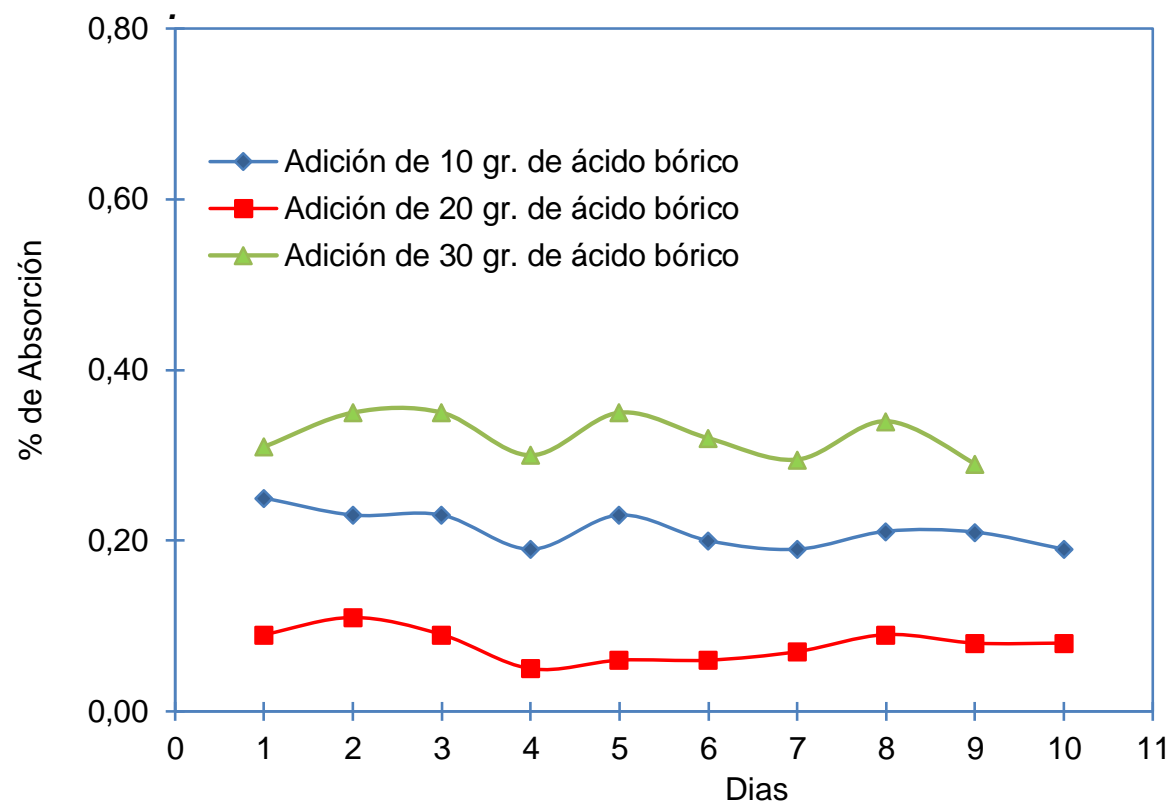

Fig. 3: Representación gráfica de la absorción (porcentajes de absorción de 10,20 y 30 gr)

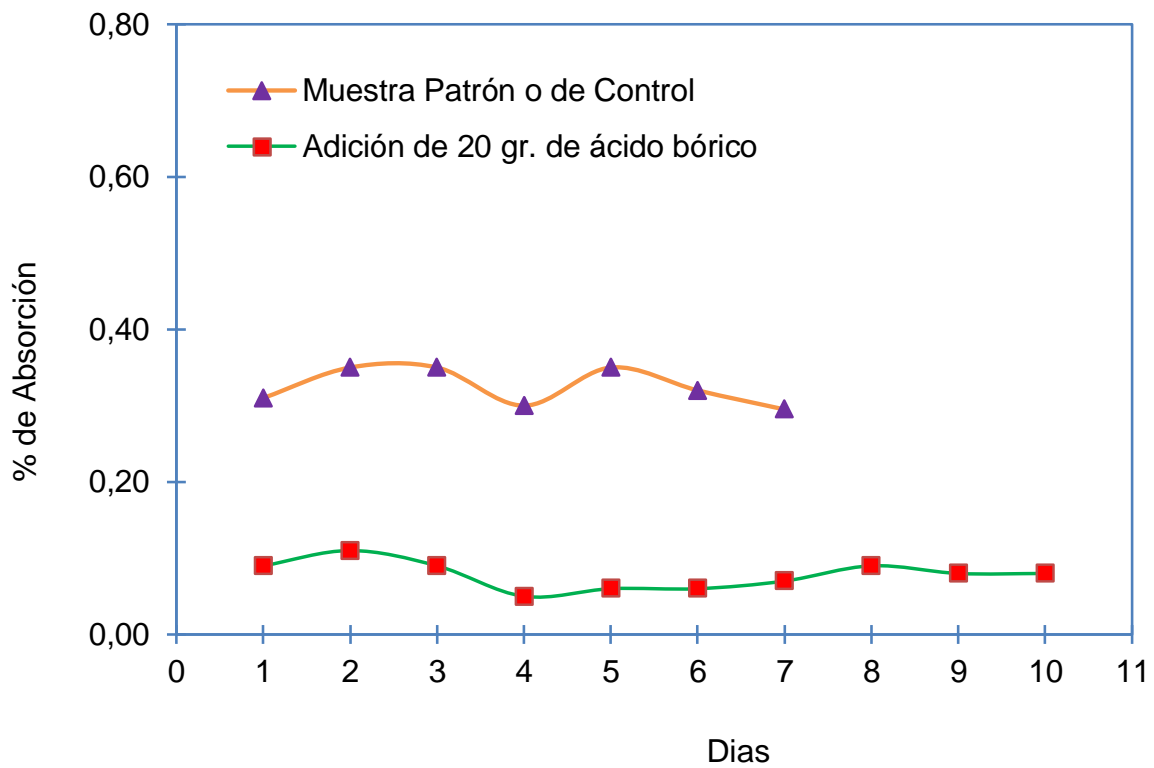

Fig. 4: Representación gráfica de la absorción (porcentajes de absorción 20 gr. Muestra Patrón)

Tabla 8: Análisis de varianza para niveles de absorción de agua y el tiempo

\begin{tabular}{|l|l|l|l|l|l|}
\hline Origen & Tipo III de suma de cuadrados & gl & Media cuadrática & F & Sig. \\
\hline Modelo & 1,460485 & 12 & 0,121707 & 497,28931 & 0,00000 \\
\hline Grupo & 0,277233 & 2 & 0,138617 & 566,38104 & 0,00000 \\
\hline Tiempo & 0,007860 & 9 & 0,000873 & 3,568332 & 0,01155 \\
\hline Error & 0,004161 & 17 & 0,000245 & & \\
\hline Total & 1,464646 & 29 & & & \\
\hline
\end{tabular}


Tabla 9: Comparaciones de promedios para los niveles de absorción de agua, según Tukey, Duncan y Sheffe

\begin{tabular}{|c|c|c|c|c|c|}
\hline \multirow[t]{2}{*}{ Grupo } & \multicolumn{2}{|l|}{$\bar{N}$} & \multicolumn{3}{|c|}{ Subconjunto } \\
\hline & & & 1 & 2 & 3 \\
\hline \multirow[t]{4}{*}{ HSD Tukey } & Grupo 2 & 10 & 0,08 & & \\
\hline & Grupo 1 & 10 & & 0,213 & \\
\hline & Grupo 3 & 9 & & & 0,3228 \\
\hline & Sig. & & 1,000 & 1,000 & 1,000 \\
\hline \multirow[t]{4}{*}{ Duncan } & Grupo 2 & 10 & 0,08 & & \\
\hline & Grupo 1 & 10 & & 0,213 & \\
\hline & Grupo 3 & 9 & & & 0,3228 \\
\hline & Sig. & & 1,000 & 1,000 & 1,000 \\
\hline \multirow[t]{4}{*}{ Scheffe } & Grupo 2 & 10 & 0,08 & & \\
\hline & Grupo 1 & 10 & & 0,213 & \\
\hline & Grupo 3 & 9 & & & 0,3228 \\
\hline & Sig. & & 1,000 & 1,000 & 1,000 \\
\hline
\end{tabular}

Tabla 10: Prueba de normalidad para los tres niveles como son los porcentajes de ácido bórico

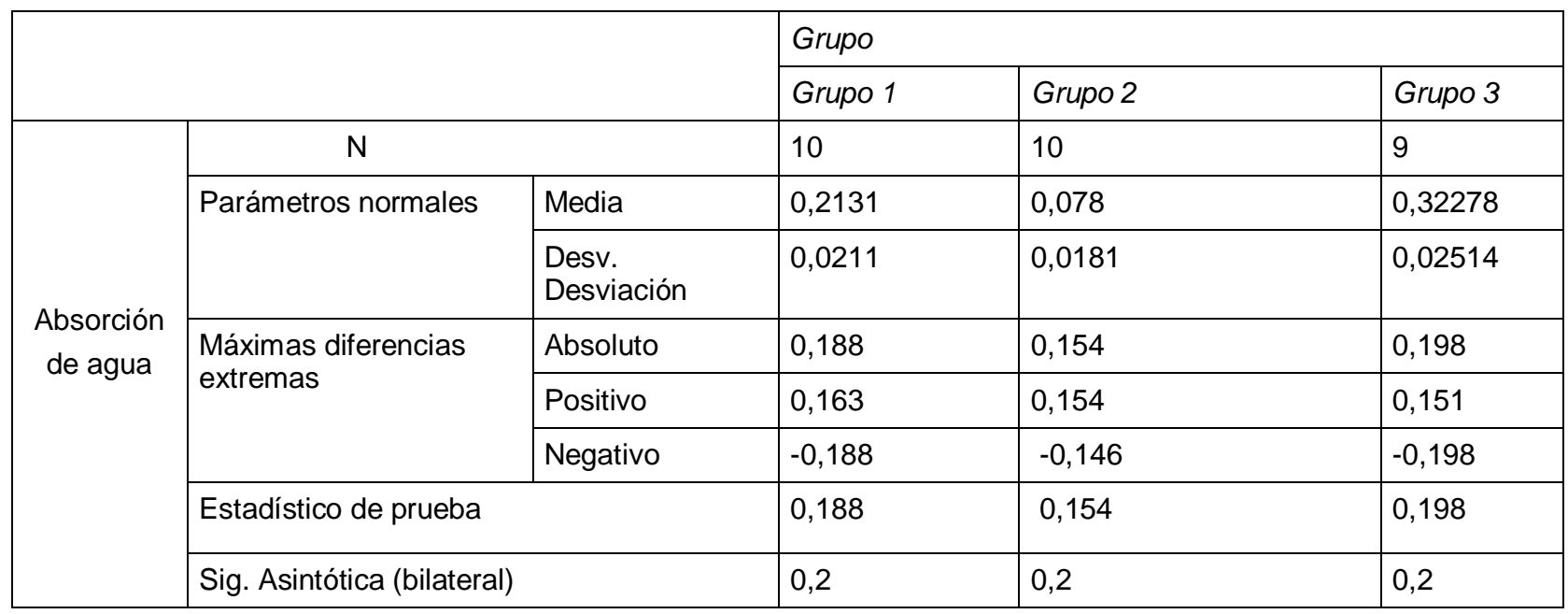

Tabla 11: Prueba de normalidad para los niveles de absorción de agua

\begin{tabular}{|c|c|c|c|c|}
\hline & & & \multicolumn{2}{|l|}{ Niveles de absorción de agua } \\
\hline & & & $\begin{array}{l}20 \mathrm{~g} \text { de ácido bórico en la mezcla } \\
\text { base }\end{array}$ & $\begin{array}{l}\text { Og de ácido } \\
\text { bórico }\end{array}$ \\
\hline \multirow{8}{*}{$\begin{array}{l}\text { Agua } \\
\text { absorbida }\end{array}$} & \multicolumn{2}{|l|}{$\mathrm{N}$} & 10 & 7 \\
\hline & \multirow[t]{2}{*}{ Parámetros normales } & Media & 0,078 & 0,12 \\
\hline & & Desv. Desviación & 0,01814 & 0,04243 \\
\hline & \multirow[t]{3}{*}{ Máximas diferencias extremas } & Absoluto & 0,154 & 0,256 \\
\hline & & Positivo & 0,154 & 0,189 \\
\hline & & Negativo & $-0,146$ & $-0,256$ \\
\hline & \multicolumn{2}{|l|}{ Estadístico de prueba } & 0,154 & 0,256 \\
\hline & \multicolumn{2}{|l|}{ Sig. asintótica(bilateral) } & 0,2 & 0,185 \\
\hline
\end{tabular}


Tabla 12: Prueba de homocedasticidad T - Student para los niveles de absorción de agua

\begin{tabular}{|c|c|c|c|c|}
\hline Suposiciones & \multicolumn{3}{|l|}{ Estadísticos } & Agua absorbida \\
\hline \multirow{9}{*}{$\begin{array}{l}\text { Se asumen } \\
\text { varianzas iguales }\end{array}$} & \multirow{2}{*}{$\begin{array}{l}\text { Prueba de Levene de } \\
\text { igualdad de varianzas }\end{array}$} & \multicolumn{2}{|l|}{$\mathrm{F}$} & 15,743 \\
\hline & & \multicolumn{2}{|l|}{ Sig. } & 0,001 \\
\hline & \multirow{7}{*}{$\begin{array}{l}\text { prueba t para la } \\
\text { igualdad de medias }\end{array}$} & \multicolumn{2}{|l|}{$\mathrm{t}$} & $-2,814$ \\
\hline & & \multicolumn{2}{|l|}{ gl } & 15 \\
\hline & & \multicolumn{2}{|l|}{ Sig. (bilateral) } & 0,013 \\
\hline & & \multicolumn{2}{|l|}{ Diferencia de medias } & $-0,042$ \\
\hline & & \multicolumn{2}{|l|}{ Diferencia de error estándar } & 0,01493 \\
\hline & & \multirow{2}{*}{$\begin{array}{l}95 \% \text { de intervalo de } \\
\text { confianza de la diferencia }\end{array}$} & Inferior & $-0,07381$ \\
\hline & & & Superior & $-0,01019$ \\
\hline \multirow{7}{*}{$\begin{array}{l}\text { No se asumen } \\
\text { varianzas iguales }\end{array}$} & \multirow{7}{*}{$\begin{array}{l}\text { prueba t para la } \\
\text { igualdad de medias }\end{array}$} & \multicolumn{2}{|l|}{$\mathrm{t}$} & $-2,466$ \\
\hline & & \multicolumn{2}{|l|}{ gl } & 7,551 \\
\hline & & \multicolumn{2}{|l|}{ Sig. (bilateral) } & 0,041 \\
\hline & & \multicolumn{2}{|l|}{ Diferencia de medias } & $-0,042$ \\
\hline & & \multicolumn{2}{|l|}{ Diferencia de error estándar } & 0,01703 \\
\hline & & \multirow[t]{2}{*}{$\begin{array}{l}95 \% \text { de intervalo de } \\
\text { confianza de la diferencia }\end{array}$} & Inferior & $-0,08168$ \\
\hline & & & Superior & $-0,00232$ \\
\hline
\end{tabular}

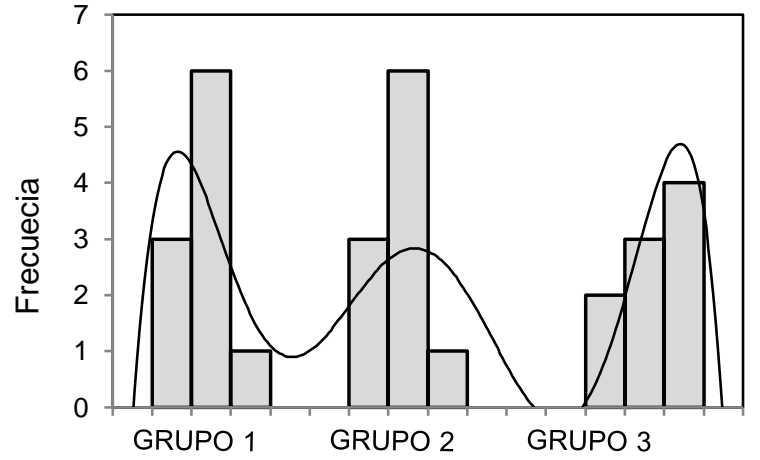

Fig. 5: Comportamiento de normalidad para los 3 niveles indicando los porcentajes de ácido bórico.

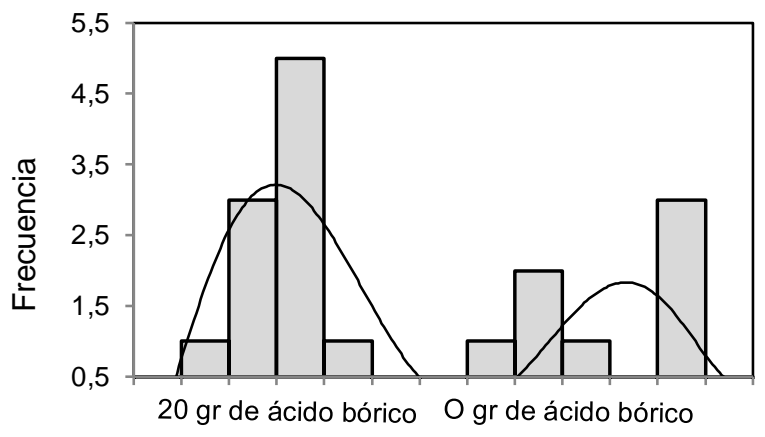

Fig.7: Comportamiento de normalidad para los niveles de absorción de agua.

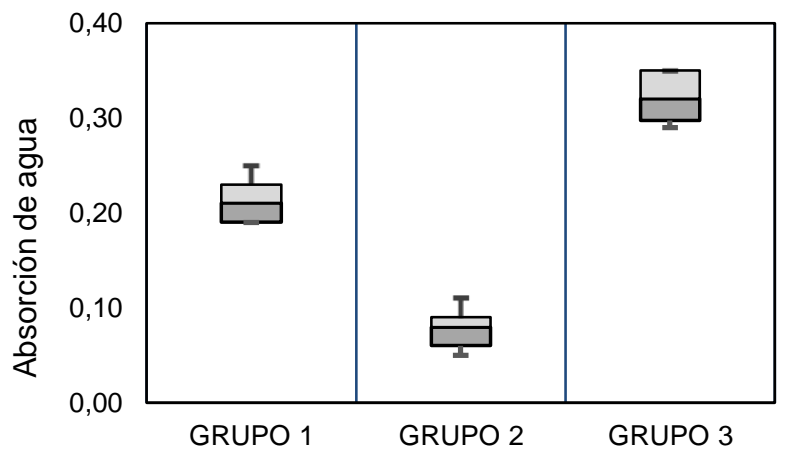

Fig. 6: Diagrama de cajas y bigotes para los 3 niveles Indicando los porcentajes de ácido bórico.

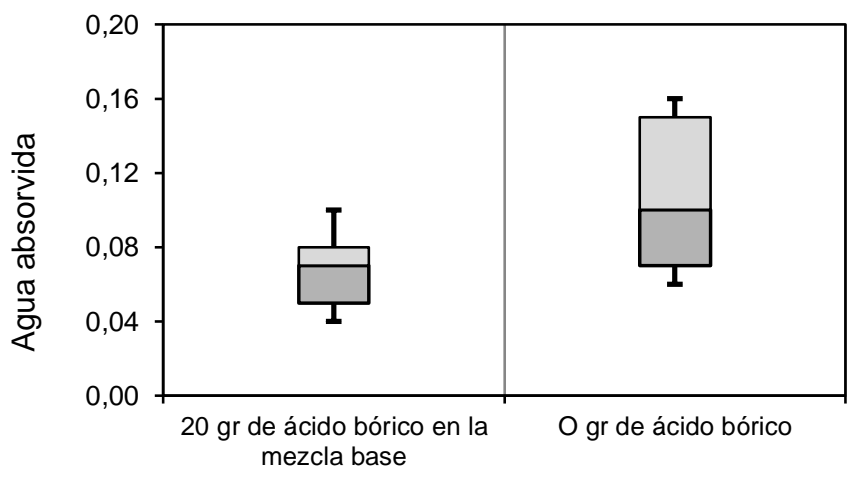

Fig.8: Diagrama de cajas para los niveles de absorción de agua.

\section{DISCUSION}

La mayoría de los recubrimientos utilizados como esmaltes cerámicos hasta hoy desarrollados, son compuestos formados por borosilicato de plomo, que incluyen también compuestos de sílice y boro, algunos pueden incluir estaño para darle mayor opacidad al producto resultante (Vicentiz, 2004). El engobe borosilocatado es un producto que se diferencia de estos recubrimientos, porque se obtiene de insumos reciclados además del boro y de sílice, significando un procedimiento más amigable con el medio ambiente. Este compuesto se caracteriza por su brillantez, pulidez y la propiedad de impermeabilidad o reducida 
absorción de fluidos como el agua. En el contexto de la literatura internacional, existen resultados relacionados a esta temática, en los que se demuestra, a diferencia de nuestro estudio, que se reduce la propiedad de durabilidad, con el transcurrir del tiempo de los compuestos vítreos, cuándo se exponen en contacto con el agua. Varshneya, y Mauro, (2019), Wang et al. (2016) y Surendranathan et al. (2014) coinciden en que el agua es un agente corrosivo, una amenaza a la durabilidad del vidrio sobre el que actúa, y más aún si es un vidrio borosilicato, porque es más débil que un vidrio silíceo. En contraposición a lo mencionado, en esta investigación, el boro, es el elemento cuya incorporación en la mezcla para preparar un esmalte, permite que sea más impermeable ante el contacto con agua, en contraste con al material de loza vitrificada del que están hechos los actuales mobiliarios sanitarios, esto se logra, diseñándose como una película delgada de borosilicato mejorado, la cual lleva incluido un porcentaje de ácido bórico del $20 \%$. El espesor de esta capa o película del esmalte vitrificante que recubre las muestras se encuentra en un rango comprendido entre $0.5 \mathrm{~mm}$ y $1.00 \mathrm{~mm}$. Se ha identificado 3 aspectos bajo los cuales se hicieron comparaciones con otros resultados similares, a saber en la metodología, el tipo de insumos empleados y la concentración de boro añadido.

En cuanto a la metodología empleada, Surendranathan et al.(2014), desarrollan un procedimiento para conseguir un material vítreo altamente resistente a la corrosión que puede provocar el agua, separando el estudio de los vidrios de silicato por fases consecutivas, proponiendo el mejoramiento de la "sustancia amorfa" de la que se ha formado el vidrio borosilicatado, a través de la adición de sucesivas porciones de compuestos boratados hasta lograr un punto preciso de máxima resistencia a la corrosión y a la humedad, tomando en cuenta las mediciones de los parámetros $\mathrm{PH}$, tiempo y temperatura, lo cual se diferencia con la presente investigación, en que los procedimientos experimentales son independientes de la temperatura bajo la cual está hechos. También Schoenfish et al. (2017), desarrollan una patente de invención, que trata de un recubrimiento hidrófobo para materiales vítreos.

En este trabajo encontramos similitudes entre los resultados logrados con nuestra experimentación y los mencionados en esta patente, relacionados a espesor de recubrimiento, tipo de material del que está hecho y la finalidad del compuesto, sin embargo desarrollan un procedimiento metodológico distinto porque se centra en obtener superficies lisas a partir de sustancias amorfas conformadas por sustancias silíceas como la silicona que pertenece al mismo grupo de materiales amorfos que nuestro compuesto vítreo, sumamente delgadas en unidades nanométricas y que son altamente hidrófobas o que rechazan toda posibilidad de absorción de agua, además de que incluyen a los boruros, los cuales se obtienen del boro como posibles insumos para su fabricación u obtención. Así también, Varshneya y Mauro, (2019), explican en los resultados de su trabajo de investigación, que los recubrimientos obtenidos son una barrera para impedir el acercamiento del fluido de ataque a la superficie del vidrio, reduciendo la concentración de álcali en la superficie que se desea proteger, puliendo al fuego un producto vidriado durante la formación. El pulido al fuego de un vidrio que contiene $\mathrm{Na}_{2} \mathrm{O}$ hace que el $\mathrm{Na}_{2} \mathrm{O}$ se volatilice, forzando a dos oxígenos no híbridos a formar un enlace puente. La superficie se vuelve rica en sílice y, en consecuencia, aumenta su durabilidad.

En cuanto al tipo de insumos utilizados, Wang et al. (2016), sustentan que el proceso de corrosión del vidrio en contacto con el agua, consiste en un complejo y lento proceso que implica una difusión e intercambio de iones en la superficie del vidrio y proponen utilizar una película delgada de grafeno, el cual se comportaría como un recubrimiento delgado, transparente, poco permeable al agua y resistente químicamente. Obteniéndose luego de ser sumergido en agua durante 120 días, que las muestras recubiertas de grafeno, comparadas con las otras muestra de vidrio sin recubrimiento, que no presentaron casi ninguna erosión ni desgaste en su superficie. Este resultado y procedimiento son muy similares al realizado en nuestra investigación, solo que se diferencia en que los investigadores utilizan el grafeno como recubrimiento y en el presente trabajo, el engobe borosilicatado.

Finalmente sobre la concentración de boro, en el trabajo realizado por Hasanuzzaman et al. (2016) se analiza y discute sobre las ventajas que tiene el vidrio poroso de borosilicato, al permitir reducir los daños por corrosión por exposición a humedad. En el texto se menciona que el "oxido de boro" o "trióxido de boro" reduce las temperaturas de fusión y de trabajo mejorando la estabilidad hidrolítica cuando se usa por debajo del 13\% en peso en la composición, lo cual es aproximadamente un $2 \%$ de presencia de Boro dentro de la misma. Utilizar cantidades por encima de esta proporción de $\mathrm{B}_{2} \mathrm{O}_{3}$ en el vidrio de borosilicato, tienen un efecto adverso sobre la resistencia al ácido y aumentan la posibilidad de formar boratos de metales alcalinos altamente volátiles; y si se utilizan las proporciones de $\mathrm{B}_{2} \mathrm{O}_{3}$ por debajo de esa misma concentración, aumentan la susceptibilidad a la cristalización del punto de fusión del vidrio al crear enlaces más seguros con iones de metales alcalinos.

En nuestra investigación, a diferencia de lo mencionado por Hasanuzzaman et al.(2016), utilizamos ácido bórico en concentraciones del $20 \%$ en peso del total del peso en seco del compuesto, combinado con otros compuestos a saber petroborato de sodio, sílice, oxido de sodio y en muy baja proporción otros óxidos de potasio, calcio y aluminio, con lo que se ha obtenido resultados positivos de reducida absorción e impermeabilidad, la presencia del boro dentro de nuestro compuesto supera el $6 \%$ del total en peso. 
Normalmente en la composición de un vidrio de borosilicato, su composición es: $70 \%$ sílice, 10\% óxido bórico, $8 \%$ óxido de sodio, $8 \%$ óxido de potasio. $1 \%$ óxido de calcio y $2 \%$ óxido de aluminio.

El engobe borosilicatado presento un punto exacto de consistencia de secado del soporte para no permitir que la contracción térmica produzca resquebrajaduras en el mismo por encogimiento (Bruguera,1985), y tiene una viscosidad esperada que es capaz de formar una interface con el mismo, significando una ventaja en relación a los procedimientos anteriores ya que se incluye el elemento toxico-contaminante, ácido bórico, en cantidades proporcionales al $20 \%$ anulando su toxicidad al entrar a un proceso de vitrificación cuando se combina con otros insumos para dar paso a una nueva sustancia diferente a sus elementos generadores, un compuesto con un bajo índice de absorción, además presenta un punto de fusión de $950^{\circ} \mathrm{C}$, lo cual resulta ventajoso, porque de esta manera se reduce el consumo de energía que frecuentemente es de $1.400^{\circ} \mathrm{C}$.

Como ha podido verse, los valores de menor absorción de agua corresponden a los especímenes con concentración de boro menores o iguales al 20\%, al incrementar a 30\% se observó una deformación de la textura del engobe, presentando porosidades muy grandes. García (2016), utilizó el ácido bórico en altas proporciones sin afectar las propiedades mínimas para que la suspensión llegue a convertirse en engobe", pues fueron superiores al 50\%, el ácido bórico se comportó como estabilizante y contribuyo al blanqueado de la suspensión arcillosa diseñada para un engobe, mientras que en nuestra propuesta, el ácido bórico contribuyo a lixiviar los compuestos minerales que integran los compuestos que dan el color, sin alterar las propiedades pastosas como engobe, permitiendo la vitrificación ahorrando la cantidad de insumos arcillosos, ya que sin alterar el peso total, se ha aumentado la proporción de fundente y se ha disminuido la proporción de plastificantes.

\section{CONCLUSIONES}

De acuerdo a los argumentos de la discusión y los resultados encontrados se concluye lo siguiente:

1.- La superficie vítrea del nuevo material propuesto es menos absorbente que la superficie de la loza vitrificada para inodoros, cuando se incluye el "ácido bórico" como insumo en porcentajes en peso del $20 \%$ dentro del diseño de mezclas para el compuesto del engobe borosilicatado.

2.- Los valores de baja absorción o impermeabilidad, se explican en primer lugar, a que el ácido bórico, que es una sustancia de bajo P.H., al actuar como fundente dentro de la pasta del engobe, disminuye la susceptibilidad a la cristalización, asegurando así ,el proceso normal hacia la vitrificación del compuesto; y en segundo lugar, una vez lograda la vitrificación, la superficie vitrificada obtenida, presenta propiedades hidrófobas, resistentes a la corrosión, a la humedad y por ende a toda posibilidad de absorción de agua.

3.- El "engobe borosilicatado", es amigable con el medio ambiente en relación a compuestos ya existentes, porque incluye además del ácido bórico, a otros insumos boratados, los cuales al ser sometidos a la vitrificación, anulan la toxicidad en conjunto, para dar paso a una sustancia totalmente diferente.

4.- En el engobe desarrollado, sin alterar los totales del peso de la mezcla en seco, se ha conseguido disminuir la proporción de plastificantes y aumentar la proporción de vitrificantes, significando un ahorro de energía porque el punto de fusión disminuye al emplear únicamente $950^{\circ} \mathrm{C}$ para la cocción.

5.- El ácido bórico se puede utilizar solo entre el rango del 10 al $20 \%$ del total del peso de la mezcla para fines de elaborar engobes con propiedades de esmalte impermeable, una concentración menor al $10 \%$ o superior al $20 \%$ no produce resultados favorables en la medición de la absorción.

6.- De los 3 resultados obtenidos en la experimentación, el que mejor apariencia tiene como engobe es el de $20 \%$, luego le sigue el de $10 \%$ y finalmente el de $30 \%$.

\section{REFERENCIAS}

ASTM C127-04, Standard Test Method for Density, Relative Density (Specific Gravity), and Absorption of Coarse Aggregate, ASTM International, West Conshohocken, PA, 2004, Pensilvania EE.UU. doi: 10.1520/C0127-04.(2004)

Aparicio, P. y Galán, E., Materias primas para la industria cerámica. Universidad de Sevilla, España. (2005)

Barba, A., Beltrán, V. y otros tres autores, Materias Primas para la Fabricación de Soportes de Baldosas Cerámicas, 2da Edición, Instituto de Tecnología Cerámica-AICE, ISBN: 8492317663, Castellón de la Plana, España (2002).

Bruguera-I, J.T, Manual práctico de cerámica. Ed. Omega, Barcelona, ISBN 13: 9788428207553. (1985).

Cardona-Restrepo, L., Estudio del mecanismo de opacificación de un recubrimiento cerámico. Tesis o trabajo de investigación presentada(o) para optar al título de: Magister en Ingeniería de Materiales y Procesos. Universidad Nacional de Colombia Facultad de Minas Medellín, Colombia (2013). 
Gomes, D.G., Garcia-Ten, J., y otros cuatro autores, Influencia de las tensiones residuales sobre el comportamiento frente al corte del gres porcelánico esmaltado, doi:10.1016/j.bsecv.2016.06.002, Boletín de la Sociedad española de cerámica y vidrio, Vol. 55(4), 143-151 (2016).

Cruz-Salgado, J., Desarrollo de un nuevo criterio para la selección de la variable de holgura en diseño de experimentos de mezclas, Tesis para obtener el grado académico de doctor en Ciencia y Tecnología en la Especialidad de Ingeniería Industrial y Manufacturera Instituto de Tecnología de Illinois, EE. UU. (2015).

De Mello, A.E., Danelon, M., y otros seis autores, In vitro effect of amorphous calcium phosphate paste applied for extended periods of time on enamel remineralization,doi:10.1590/1678-7757-2016-0513 Journal of Applied Oral Science Vol.25 (6), 596-603 (2017).

García-Acevedo, S., Estudio de la capacidad suspensionante de componentes arcillosos en engobes cerámicos. Tesis presentada para optar al título de Maestría en Ingeniería, Materiales y Proceso, Universidad Nacional de Colombia, Facultad de Minas, Medellín, Colombia (2016).

Hasanuzzaman, M., Ghani, O., y otros tres autores, Chapter Production and Treatment of Porous Glass Materials for Advanced Usage, Reference Module in Materials Science and Materials Engineering, Ed. Hashmi, Saleem. Elsevier, Pages 1-13, doi:10.1016/B978-0-12-803581-8.03999-0,Dublin Ireland (2016).

Hernández. M., Suarez.G. y otros cuatro autores, Formation, microstructure and properties of aluminum borate ceramics obtained from alumina and boric acid, https://doi.org/10.1016/j.ceramint.2016.11.002, Ceramics International, Vol. 43(2), 2188-2195 (2017).

Ministerio de Energía y Minas "MINEM". "Arsénico y boro en el Rio Tambo".Energiminas” Energía - Minería-Perú [serie en internet]. noviembre 2018, Vol. (03), No 75 (2018).

Mehta, J., Kumar, V., y Gupta P., Role of Thermal Spray Coatings on Wear, Erosion and Corrosion Behavior: A Review, doi:10.6180/jase.2017.20.4.05, Applied Science and Engineering, Vol.(204), 445-452 (2017).

Saad. B.H., F., Chapter 01 Overview. Bioceramics: For Materials Science and Engineering, Woodhead, Publishing Series in Biomaterials, 1st Edition, Woodhead Publishing, 1-37, https://doi.org/10.1016/B978-0-08-102233-7.00001-X, United States (2019).

Schoenfish, M.H., Privett, B., Ho Shen,J.,y Youn,J., Superhydrophobic coatings and methods for their preparation, Estados Unidos Patent No USO0 9675994 B2, 01 de Junio del 2012 (2017).

Surendranathan, A.O., Corrosion of ceramics and refractories, 1st Edition, 74-75, CRC Press, London, United Kingdom (2014).

Thompson, W. y otros cuatro autores, Systematic study of sol-gel parameters on $\mathrm{TiO}_{2}$ coating for $\mathrm{CO}_{2}$ photoreduction", https://doi.org/10.1016/j.apcatb.2018.07.018, Applied Catalysis B: Environmental Vol.(238), 136-146 (2018).

Varshneya, Arun K. y Mauro J. C., Chapter 17 Chemical durability, Fundamentals of Inorganic Glasses, Third Edition, Pages. 473-486, https://doi.org/10.1016/C2017-0-04281-7, EE.UU. (2019)

Vicentiz, S.L., Materiales cerámicos., Materias primas, programa de suministro. Pesos atómicos de los elementos químicos. Análisis químicos. Valladolid, España (2018).

Wandscher V.F., Fraga S., y otros cinco autores, Tribochemical Glass Ceramic Coating as a New Approach for Resin Adhesion to Zirconia, doi:10.3290/j.jad.a36892, J Adhes Dent., Vol 18(5), 435-440 (2016).

Wang, B., Cunning, B., y otros cuatro autores, Graphene Coatings as Barrier Layers to Prevent the Water-Induced Corrosion of Silicate Glass, https://doi.org/10.1021/acsnano.6b04363, AS Nano, Vol.10(11), 9794-9800 (2016).

Zanelli, Ch., Dominguez, E., y otros seis autores., "Recycling of residual boron muds into ceramic tiles". doi:10.1016/j.bsecv.2019.01.002, Boletín de la sociedad española de cerámica doi y vidrio. Vol. 58(5), 199-210 (2019). 
